\title{
Numerically Efficient Fourier-Based Technique for Calculating Error Probabilities with Intersymbol Interference
}

\author{
Michael Reuter
}

\begin{abstract}
We propose a numerically efficient technique for calculating the probability of symbol error for arbitrary coherent modulation schemes in the presence of intersymbol interference (ISI) and additive noise. The probability of error is formulated in terms of an inverse Fourier transform of the windowed characteristic function of the random variable representing the interfering symbols and the noise process. The integral is evaluated numerically using the sampling theorem.
\end{abstract}

Index Terms - Intersymbol interference, probability of error.

\section{INTRODUCTION}

$\mathbf{T}$ HE EFFECTS of intersymbol interference (ISI) can degrade the probability of error $\left(P_{\mathrm{E}}\right)$ performance of digital receivers [1]. One technique for determining the performance of the receiver is to evaluate $P_{\mathrm{E}}$ conditioned on a particular sequence of ISI symbols and then average over all possible sequences. This exact technique can be prohibitively expensive computationally, and so a considerable amount of work has been done on numerically efficient methods for evaluating or bounding $P_{\mathrm{E}}$. A summary of many of these techniques is given in [2].

Although recent advances are numerically accurate and easy to implement in computer-aided design (CAD) tools [2]-[5], they have dealt with one-dimensional (1-D) or real coherent signaling. While the algorithms presented in [6] and [7] are applicable to two-dimensional (2-D) formats, they have only been applied to $M$-ary phase-shift keying ( $M$ PSK) and $M$-ary quadrature amplitude modulation ( $M$-QAM), respectively.

We present a general method which can be applied to arbitrary 1- or 2-D coherent signaling formats. We demonstrate that the probability of correctly decoding a particular symbol is the inverse Fourier transform of the windowed characteristic function of the random variable representing the interfering symbols and the additive noise component evaluated at a point corresponding to the decision region of the symbol. We use the sampling theorem and appropriate spectral windowing and truncation to evaluate the integral numerically.

Paper approved by R. Kennedy, the Editor for Data Communications Modulation \& Signal Design of the IEEE Communications Society. Manuscript received December 2, 1995; revised October 30, 1996. This work was supported by the Naval Command, Control and Ocean Surveillance Center RDT\&E Division Independent Research Program.

The author is with the Naval Command, Control and Ocean Surveillance Center RDT\&E Division, Code D855, San Diego, CA 92152 USA and with the Department of Electrical and Computer Engineering, University of California at San Diego, La Jolla, CA 92093 USA.

Publisher Item Identifier S 0090-6778(97)04181-0.

\section{Problem Statement and General Solution}

Consider the sampled output $y$ of a coherent receiver. In the presence of ISI and additive noise, $y$ is given by

$$
y=\sum_{\substack{k=-N \\ k \neq 0}}^{N} b_{k} a_{k}+b_{0} a_{0}+n .
$$

The $b_{k}$ 's represent the transmitted data sequence in which each element is one of $M$-possible equally probable symbols, the $a_{k}$ 's are the ISI coefficients, $b_{0}$ is the current symbol to be detected, and $n$ is the noise. We assume a finite number $N$ of symbols before and after $b_{0}$. The $b_{k}$ 's are assumed to be mutually independent and independent of the noise. In general, all components of the equation are complex.

The probability of making a correct decision given that the $j^{\text {th }}$ symbol $s_{j}$ is sent is

$$
P_{\mathrm{C} j}=\operatorname{Pr}\left(y \in D_{j} \mid b_{0}=s_{j}\right)
$$

where $D_{j}$ is the decision region in the complex plane corresponding to $s_{j}$. The probability of error is given by

$$
P_{\mathrm{E}}=1-\frac{1}{M} \sum_{j=1}^{M} P_{\mathrm{C} j} .
$$

The validity of (2) is predicated on the assumption that the variate $y$ can be directly applied to the decision device to achieve the desired error performance. In practice, if the effects of ISI and the coefficient $\alpha_{0}$ are too severe, the decision region $D_{j}$ is modified or equalization is used to mitigate these effects.

The exact technique for calculating $P_{\mathrm{C} j}$ evaluates the probability of a correct decision by conditioning $y$ on a particular sequence of interfering symbols. The resulting variate has the same distribution as the noise. Then $P_{\mathrm{C} j}$ is determined by averaging over all possible interference sequences. However, the computational cost can be prohibitively large because there are $M^{2 N}$ possible interference sequences.

In the proposed method, we incorporate ISI more efficiently by working on the characteristic function of the variate $y$ conditioned only on $b_{0}=s_{j}$. Writing $y$ in terms of its in-phase and quadrature components as $y=y_{\mathrm{I}}+i y_{\mathrm{Q}}$, we denote the bivariate characteristic function of $y$ as $h_{Y}\left(\omega_{\mathrm{I}}, \omega_{\mathrm{Q}}\right)$. Conditioning on $b_{0}=s_{j}$, we get

$$
\begin{aligned}
& h_{Y}\left(\omega_{\mathrm{I}}, \omega_{\mathrm{Q}}\right)= \\
& \quad h_{\mathrm{ISI}}\left(\omega_{\mathrm{I}}, \omega_{\mathrm{Q}}\right) h_{\mathrm{N}}\left(\omega_{\mathrm{I}}, \omega_{\mathrm{Q}}\right) e^{i\left(\omega_{\mathrm{I}} \operatorname{Re}\left\{s_{j} a_{0}\right\}+\omega_{\mathrm{Q}} \operatorname{Im}\left\{s_{j} a_{0}\right\}\right)}
\end{aligned}
$$

U.S. Government work not protected by U.S. copyright. 
where

$$
h_{\mathrm{ISI}}\left(\omega_{\mathrm{I}}, \omega_{\mathrm{Q}}\right)=\prod_{\substack{k=-N \\ k \neq 0}}^{N} h_{\mathrm{ISI} k}\left(\omega_{\mathrm{I}}, \omega_{\mathrm{Q}}\right)
$$

is the characteristic function of the variate representing the interfering symbols, and $h_{\mathrm{N}}\left(\omega_{\mathrm{I}}, \omega_{\mathrm{Q}}\right)$ is the characteristic function of the noise process. The probability density function of $y$ is given as

$$
\begin{aligned}
& f_{Y}\left(y_{\mathrm{I}}, y_{\mathrm{Q}}\right)= \\
& \quad \frac{1}{(2 \pi)^{2}} \int_{-\infty}^{\infty} \int_{-\infty}^{\infty} h_{Y}\left(\omega_{\mathrm{I}}, \omega_{\mathrm{Q}}\right) e^{-i\left(\omega_{\mathrm{I}} y_{\mathrm{I}}+\omega_{\mathrm{Q}} y_{\mathrm{Q}}\right)} d \omega_{\mathrm{I}} d \omega_{\mathrm{Q}} .
\end{aligned}
$$

Then, because

$$
P_{\mathrm{C} j}=\iint_{D_{j}} f_{Y}\left(y_{\mathrm{I}}, y_{\mathrm{Q}}\right) d y_{\mathrm{I}} d y_{\mathrm{Q}}
$$

and assuming that $h_{Y}\left(\omega_{\mathrm{I}}, \omega_{\mathrm{Q}}\right)$ is absolutely integrable, we use (6) in (7) and interchange the order of integration to get $P_{\mathrm{C} j}$, shown in (8) at the bottom of the page, where $K_{j}\left(\omega_{\mathrm{T}}, \omega_{\mathrm{Q}}\right)$ is the Fourier transform of the decision region $D_{j}$. This equation is an inverse Fourier transform evaluated at $\left(\operatorname{Re}\left\{s_{j} a_{0}\right\}, \operatorname{Im}\left\{s_{j} a_{0}\right\}\right)$.

It is instructive to explicitly state the equivalence of the probability domain, indexed by $\left(t_{\mathrm{I}}, t_{\mathrm{Q}}\right)$, and the spectral domain, indexed by $\left(\omega_{\mathrm{I}}, \omega_{\mathrm{Q}}\right)$, with the following relations:

$$
P_{j}\left(t_{\mathrm{I}}, t_{\mathrm{Q}}\right)=\mathcal{F}^{-1}\left\{K_{j}\left(\omega_{\mathrm{I}}, \omega_{\mathrm{Q}}\right) h_{\mathrm{ISI}}\left(\omega_{\mathrm{I}}, \omega_{\mathrm{Q}}\right) h_{\mathrm{N}}\left(\omega_{\mathrm{I}}, \omega_{\mathrm{Q}}\right)\right\}
$$

or equivalently

$$
P_{j}\left(t_{\mathrm{I}}, t_{\mathrm{Q}}\right)=D_{j}\left(t_{\mathrm{I}}, t_{\mathrm{Q}}\right) * f_{\mathrm{ISI}}\left(t_{\mathrm{I}}, t_{\mathrm{Q}}\right) * f_{\mathrm{N}}\left(t_{\mathrm{I}}, t_{\mathrm{Q}}\right)
$$

where $f_{\mathrm{ISI}}\left(t_{\mathrm{I}}, t_{\mathrm{Q}}\right)$ and $f_{\mathrm{N}}\left(t_{\mathrm{I}}, t_{\mathrm{Q}}\right)$ are the probability density functions of the random variables representing the ISI terms and the noise, respectively, and $*$ denotes convolution. $f_{\mathrm{ISI}}\left(t_{\mathrm{I}}, t_{\mathrm{Q}}\right)$ is a 2-D sequence of Dirac delta functions in which each of the $M^{2 N}$ components corresponds to a particular realization of ISI symbols. $P_{\mathrm{C} j}$ is equal to $P_{j}\left(t_{\mathrm{I}}, t_{\mathrm{Q}}\right)$ evaluated at $t_{\mathrm{I}}=\operatorname{Re}\left\{s_{j} a_{0}\right\}$ and $t_{\mathrm{Q}}=\operatorname{Im}\left\{s_{j} a_{0}\right\}$.

Several established methods exist for computing Fourier integrals [8]. The basis of the proposed technique is to evaluate (8) numerically by spectrally sampling the windowed characteristic function in (9). We use the sampling theorem and the relations given by (9) and (10) to determine the adequate resolution. By sampling with a periodic rectangular grid with spectral resolution $\Delta \omega_{\mathrm{I}}=\Delta \omega_{\mathrm{Q}}=2 \pi / T$, we get Poisson's sum [9]

$$
\begin{gathered}
\sum_{m_{\mathrm{Q}}=-\infty}^{\infty} \sum_{m_{\mathrm{I}}=-\infty}^{\infty} P_{j}\left(t_{\mathrm{I}}-m_{\mathrm{I}} T, t_{\mathrm{Q}}-m_{\mathrm{Q}} T\right) \\
=\frac{1}{T^{2}} \sum_{l_{\mathrm{Q}}=-\infty}^{\infty} \sum_{l_{\mathrm{I}}=-\infty}^{\infty} K_{j}\left(\frac{2 \pi}{T} l_{\mathrm{I}}, \frac{2 \pi}{T} l_{\mathrm{Q}}\right) \\
\cdot h_{\mathrm{ISI}}\left(\frac{2 \pi}{T} l_{\mathrm{I}}, \frac{2 \pi}{T} l_{\mathrm{Q}}\right) h_{\mathrm{N}}\left(\frac{2 \pi}{T} l_{\mathrm{I}}, \frac{2 \pi}{T} l_{\mathrm{Q}}\right) \\
\cdot e^{i\left(l_{\mathrm{I}} t_{\mathrm{I}}+l_{\mathrm{Q}} t_{\mathrm{Q}}\right) 2 \pi / T} .
\end{gathered}
$$

$T$ must be large enough so that when the left-hand side (LHS) of this equation is evaluated for $t_{\mathrm{I}}=\operatorname{Re}\left\{s_{j} a_{0}\right\}$ and $t_{\mathrm{Q}}=\operatorname{Im}\left\{s_{j} a_{0}\right\}$, the error due to aliasing has an acceptable value.

However, (11) is not defined for some decision regions. This can be seen from (10). Defining the duration $d_{f}$ of a function $f\left(t_{\mathrm{I}}, t_{\mathrm{Q}}\right)$ as $[10]$

$$
d_{f}=\int_{-\infty}^{\infty} \int_{-\infty}^{\infty}\left|t_{\mathrm{I}}\right|^{2}\left|t_{\mathrm{Q}}\right|^{2}\left|f\left(t_{\mathrm{I}}, t_{\mathrm{Q}}\right)\right|^{2} d t_{\mathrm{I}} d t_{\mathrm{Q}}
$$

we can see that although the convolution of the probability density functions $f_{\mathrm{ISI}}\left(t_{\mathrm{I}}, t_{\mathrm{Q}}\right) * f_{\mathrm{N}}\left(t_{\mathrm{I}}, t_{\mathrm{Q}}\right)$ produces a function of finite duration in the $\left(t_{\mathrm{I}}, t_{\mathrm{Q}}\right)$ plane, the decision region $D_{j}\left(t_{\mathrm{I}}, t_{\mathrm{Q}}\right)$ may be of infinite duration in the $\left(t_{\mathrm{I}}, t_{\mathrm{Q}}\right)$ plane, resulting in $P_{j}\left(t_{\mathrm{I}}, t_{\mathrm{Q}}\right)$ also being of infinite duration. Then the spectral term in (9) cannot be adequately sampled. To solve this problem, we simply limit the duration of the decision region by multiplying $D_{j}\left(t_{\mathrm{I}}, t_{\mathrm{Q}}\right)$ with an ideal brick-wall window, producing a modified decision region $\widehat{D}_{j}\left(t_{\mathrm{I}}, t_{\mathrm{Q}}\right)$ with Fourier transform $\widehat{K}_{j}\left(\omega_{\mathrm{I}}, \omega_{\mathrm{Q}}\right)$.

Finally, we truncate the spectral sum in (11). Then, the calculated probability of correctly detecting the $j$ th symbol is

$$
\begin{aligned}
\widehat{P}_{\mathrm{C} j}= & \\
& \frac{1}{T^{2}} \sum_{l_{\mathrm{Q}}=-P}^{P} \sum_{l_{\mathrm{I}}=-P}^{P} \widehat{K}_{j}\left(\omega l_{\mathrm{I}}, \omega l_{\mathrm{Q}}\right) \prod_{\substack{k=-N \\
k \neq 0 \\
\text { ISI }}}^{N}\left(\omega l_{\mathrm{I}}, \omega l_{\mathrm{Q}}\right) \\
& \cdot h_{\mathrm{N}}\left(\omega l_{\mathrm{I}}, \omega l_{\mathrm{Q}}\right) e^{i\left(\omega l_{\mathrm{I}} \operatorname{Re}\left\{s_{j} a_{0}\right\}+\omega l_{\mathrm{Q}} \operatorname{Im}\left\{s_{j} a_{0}\right\}\right)}
\end{aligned}
$$

where $\omega=2 \pi / T$ and $P_{\mathrm{C} j}=\widehat{P}_{\mathrm{C} j}+$ error.

The total numerical error in this approach can be written as error $=\epsilon_{1}-\epsilon_{2}+\epsilon_{3}$. The first component, $\epsilon_{1}$, is the result of using a windowed or modified decision region $\widehat{D}_{j}\left(t_{\mathrm{I}}, t_{\mathrm{Q}}\right)$ and can be written as

$$
\epsilon_{1}=\left(D_{j}\left(t_{\mathrm{I}}, t_{\mathrm{Q}}\right)-\widehat{D}_{j}\left(t_{\mathrm{I}}, t_{\mathrm{Q}}\right)\right) * f_{\mathrm{ISI}}\left(t_{\mathrm{I}}, t_{\mathrm{Q}}\right) * f_{\mathrm{N}}\left(t_{\mathrm{I}}, t_{\mathrm{Q}}\right)
$$

evaluated at $t_{\mathrm{I}}=a_{0 \mathrm{I}}-a_{0 \mathrm{Q}}$ and $t_{\mathrm{Q}}=a_{0 \mathrm{I}}+a_{0 \mathrm{Q}}$. The second, $\epsilon_{2}$, is due to aliasing and is determined by applying $\widehat{D}_{j}\left(t_{\mathrm{I}}, t_{\mathrm{Q}}\right)$

$$
P_{\mathrm{C} j}=\frac{1}{(2 \pi)^{2}} \int_{-\infty}^{\infty} \int_{-\infty}^{\infty} K_{j}\left(\omega_{\mathrm{I}}, \omega_{\mathrm{Q}}\right) h_{\mathrm{ISI}}\left(\omega_{\mathrm{I}}, \omega_{\mathrm{Q}}\right) h_{\mathrm{N}}\left(\omega_{\mathrm{I}}, \omega_{\mathrm{Q}}\right) e^{i\left(\omega_{\mathrm{I}} \operatorname{Re}\left\{s_{j} a_{0}\right\}+\omega_{\mathrm{Q}} \operatorname{Im}\left\{s_{j} a_{0}\right\}\right)} d \omega_{\mathrm{I}} d \omega_{\mathrm{Q}}
$$


to (10) and using (11), given by

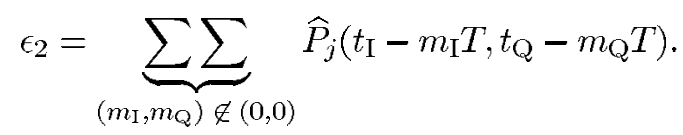

The third, $\epsilon_{3}$, is due to spectral truncation and can be bounded as shown in (16) at the bottom of the page. The proper windowing, spectral sampling resolution, and spectral truncation must be determined so that $\mid$ error $\mid \ll P_{\mathrm{C} j}$.

\section{PROBABILITY OF ERROR FOR QPSK}

To demonstrate the method, we present the results for QPSK. Let the $b_{k}$ 's in (1) represent symbols in which the inphase and quadrature components take values +1 and -1 with equal probability $\left(b_{k}= \pm 1 \pm i\right)$. The characteristic function of the $k$ th interference component $(k \neq 0)$ is given by

$$
h_{\mathrm{ISI} k}\left(\omega_{\mathrm{I}}, \omega_{\mathrm{Q}}\right)=\cos \left(\omega_{\mathrm{I}} a_{k \mathrm{I}}+\omega_{\mathrm{Q}} a_{k \mathrm{Q}}\right) \cos \left(\omega_{\mathrm{I}} a_{k \mathrm{Q}}-\omega_{\mathrm{Q}} a_{k \mathrm{I}}\right) \text {. }
$$

We assume the in-phase and quadrature components of the noise are independent and identically distributed zero-mean Gaussian processes so that

$$
h_{\mathrm{N}}\left(\omega_{\mathrm{I}}, \omega_{\mathrm{Q}}\right)=\exp \left(-\left(\omega_{\mathrm{I}}^{2}+\omega_{\mathrm{Q}}^{2}\right) \sigma^{2} / 2\right) \text {. }
$$

From (2), $P_{\mathrm{C} 1}$ is given by

$$
P_{\mathrm{C} 1}=\operatorname{Pr}\left(y \in D_{1}\left(t_{\mathrm{I}}, t_{\mathrm{Q}}\right) \mid b_{0 \mathrm{I}}=1, b_{0 \mathrm{Q}}=1\right)
$$

where $D_{1}\left(t_{\mathrm{I}}, t_{\mathrm{Q}}\right)$ is the first quadrant in the complex plane, and we have assumed that $s_{1} a_{0} \in D_{1}\left(t_{\mathrm{I}}, t_{\mathrm{Q}}\right)$. We use the modified decision region

$$
\widehat{D}_{1}\left(t_{\mathrm{I}}, t_{\mathrm{Q}}\right)= \begin{cases}1, & 0 \leq t_{\mathrm{I}}, t_{\mathrm{Q}} \leq T / 2 \\ 0, & \text { elsewhere }\end{cases}
$$

with Fourier transform

$$
\widehat{K}_{1}\left(\omega_{\mathrm{I}}, \omega_{\mathrm{Q}}\right)=\frac{T^{2}}{4} \operatorname{sinc}\left(\frac{\omega_{\mathrm{I}} T}{4 \pi}\right) \operatorname{sinc}\left(\frac{\omega_{\mathrm{Q}} T}{4 \pi}\right) e^{-i\left(\omega_{\mathrm{I}}+\omega_{\mathrm{Q}}\right) T / 4}
$$

Next, we define the term

$$
\gamma=\sum_{k=-N}^{N}\left(\left|a_{k \mathrm{I}}\right|+\left|a_{k \mathrm{Q}}\right|\right)
$$

and the user-specified parameter

$$
\epsilon=\Phi((-T / 2+\gamma) / \sigma)
$$

where $\Phi(x)=(\sqrt{2 \pi})^{-1 / 2} \int_{-\infty}^{x} \exp \left(-y^{2} / 2\right) d y$. Also, the spectral truncation index $P$ is written as a function of the variable $L$ as

$$
P=\left\lceil\frac{L T}{2 \pi \sigma}\right\rceil
$$

where $\lceil x\rceil$ denotes the least integer greater than or equal to $x$.

We can use (14)-(16) to show that if $\epsilon$ is bounded as $\epsilon<\Phi(-1), T$ is determined from (23), and $P$ and $L$ are chosen such that

$$
4\left(3+\frac{2 P}{L}\right) \frac{\Phi(-L)}{L} \leq \epsilon
$$

then the total numerical error can be bounded as

$$
\mid \text { error } \mid<30 \epsilon \text {. }
$$

Then, writing the probability of error as $P_{\mathrm{E}}=1-P_{\mathrm{C} 1}$, the calculated probability of symbol error for QPSK is given by (27), shown at the bottom of the page, where $P_{\mathrm{E}}=\widehat{P}_{\mathrm{E}}-$ error and $\omega=2 \pi / T$. So $\epsilon$ must be chosen such that $\epsilon \ll P_{\mathrm{E}}$.

We can use these results to determine the probability of error for binary phase-shift keying (BPSK) by letting $\operatorname{Im}\left\{a_{k}\right\}=0$ in (1). Then there is no crosstalk and the in-phase and quadrature components are statistically independent. The probability of bit error for BPSK can then be written as $P_{\mathrm{B}}=1-\sqrt{1-P_{\mathrm{E}}}$. Straightforward manipulations of (27) result in the calculated probability of bit error, which is identical in form to Beaulieu's equation (41a) in [3].

\section{NUMERICAL EXAMPLES}

We calculate the probability of error for QPSK with the pulse

$$
a(t)=(1+i r) \operatorname{sinc}\left(t / T_{S}\right)
$$

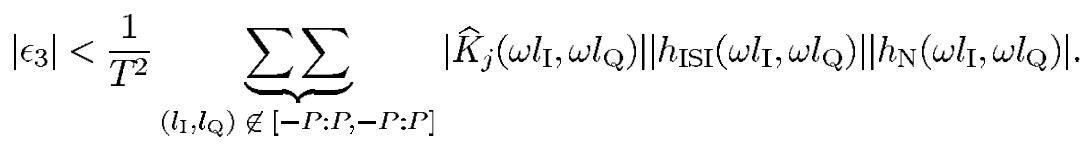

$$
\begin{gathered}
\widehat{P}_{\mathrm{E}}=1-\frac{1}{4} \underbrace{\sum_{l_{\mathrm{Q}}=-P} \sum_{l_{\mathrm{I}}=-P}^{P} \operatorname{sinc}\left(\frac{l_{\mathrm{I}}}{2}\right) \operatorname{sinc}\left(\frac{l_{\mathrm{Q}}}{2}\right) \prod_{\substack{k=-N \\
k \neq 0}}^{N} h_{\mathrm{ISI} k}\left(\omega l_{\mathrm{I}}, \omega l_{\mathrm{Q}}\right) h_{\mathrm{N}}\left(\omega l_{\mathrm{I}}, \omega l_{\mathrm{Q}}\right)}_{l_{\mathrm{I}}, l_{\mathrm{Q}} \text { odd }} \\
\cdot \cos \left(\omega l_{\mathrm{I}}\left(a_{\mathrm{OI}}-a_{\mathrm{OQ}}-T / 4\right)+\omega l_{\mathrm{Q}}\left(a_{\mathrm{OI}}+a_{\mathrm{OQ}}-T / 4\right)\right)
\end{gathered}
$$


TABLE I

QPSK ERROR PROBABILITIES FOR $t / T_{S}=0.1$,

$\mathrm{SNR}=14.98235 \mathrm{~dB}, r=0$, AND $L=7.2$

\begin{tabular}{rcccc}
\hline \hline$N$ & $T$ & $\hat{\Gamma}_{\mathrm{E}}$ & Relative Error & Relative Error Bound \\
\hline 1 & 4.8111003 & $2.6654431 \times 10^{-6}$ & $6.2562056 \times 10^{-7}$ & $3.7517237 \times 10^{-5}$ \\
2 & 5.0083197 & $8.8222171 \times 10^{-6}$ & $4.9091744 \times 10^{8}$ & $1.1335020 \times 10^{-5}$ \\
3 & 5.1396165 & $1.4551146 \times 10^{-5}$ & $8.5758927 \times 10^{-9}$ & $6.8723108 \times 10^{-6}$ \\
4 & 5.2380412 & $1.9005067 \times 10^{-5}$ & $2.3658970 \times 10^{-9}$ & $5.2617547 \times 10^{-6}$ \\
& & & & \\
10 & 5.5708367 & $3.1407996 \times 10^{-5}$ & & \\
100 & 6.4594314 & $4.2844639 \times 10^{-5}$ & & \\
1000 & 7.3636225 & $4.4205987 \times 10^{-5}$ & \\
\hline
\end{tabular}

TABLE II

QPSK ERROR PROBABILITIES FOR $t / T_{S}=0.1$,

$\mathrm{SNR}=14.98235 \mathrm{~dB}, r=0.25$, AND $L=7.2$

\begin{tabular}{rcccc}
\hline \hline$N$ & $T$ & $\hat{P}_{\mathrm{E}}$ & Relative Error & Relative Error Bound \\
\hline 1 & 5.4022729 & $3.8765087 \times 10^{-4}$ & $6.9651912 \times 10^{-10}$ & $2.5796408 \times 10^{-7}$ \\
2 & 5.6487971 & $7.4307113 \times 10^{-4}$ & $4.4673608 \times 10^{-11}$ & $1.3457662 \times 10^{-7}$ \\
3 & 5.8129181 & $9.5683016 \times 10^{-4}$ & $4.6412543 \times 10^{-12}$ & $1.0451176 \times 10^{-7}$ \\
4 & 5.9359489 & $1.0923858 \times 10^{-3}$ & $1.9310245 \times 10^{-12}$ & $9.1542749 \times 10^{-8}$ \\
& & & & \\
10 & 6.3519433 & $1.3991347 \times 10^{-3}$ & & \\
100 & 7.4626867 & $1.6308787 \times 10^{-3}$ & & \\
1000 & 8.5929256 & $1.6563448 \times 10^{-3}$ & & \\
\hline \hline
\end{tabular}

where $a_{k}=a\left(t-k T_{S}\right)$ and $t / T_{S}=0.1$. The $r$ factor induces crosstalk between the in-phase and quadrature channels. The additive noise is Gaussian with SNR defined as SNR = $|\operatorname{Re}\{a(0)\}|^{2} / \sigma^{2}$ to stay consistent with [2], [3], and SNR = 14.98235. The parameters $\epsilon, T$, and $L$ are chosen so that |error $\mid<10^{-10}$ for all cases.

Table I contains the calculated probability of symbol error $\widehat{P}_{\mathrm{E}}$ for $r=0$ and for various $N$. The relative error, which is defined as $\left|\left(P_{\mathrm{E}}-\widehat{P}_{\mathrm{E}}\right) / P_{\mathrm{E}}\right|$ [11], is also tabulated for $N=1$ to 4 , along with the estimated bound on the relative error given by $10^{-10} / P_{\mathrm{E}}$. The exact value $P_{\mathrm{E}}$ is calculated by averaging over all permutations of the interfering symbols. If the relative error is approximately $10^{-n}$, then $\widehat{P}_{\mathrm{E}}$ and $P_{\mathrm{E}}$ agree to about $n$ significant figures [11]. It can be seen that the bound |error $\mid<10^{-10}$ for this example is fairly loose because we are afforded approximately two to five more significant figures in practice than are indicated by the bound. In this example, the probability of bit error for BPSK is $P_{\mathrm{B}}=1-\sqrt{1-P_{\mathrm{E}}}$. Then selected terms in Table I agree with the corresponding terms in Table III of [3]. Table II contains the results for $r=0.25$. The degradation in performance due to crosstalk is also evident in Fig. 1 where $\widehat{P}_{\mathrm{E}}$ is plotted versus $r$ with $N=100$.

\section{CONCLUSION}

We have presented a simple, numerically efficient technique for calculating the probability of error with ISI and additive noise for arbitrary 2-D coherent modulation formats. The method requires the Fourier transform of the decision regions of the modulation scheme and the characteristic functions of the symbol variate and additive noise process. This technique is a 2-D generalization of Beaulieu's real-signaling method

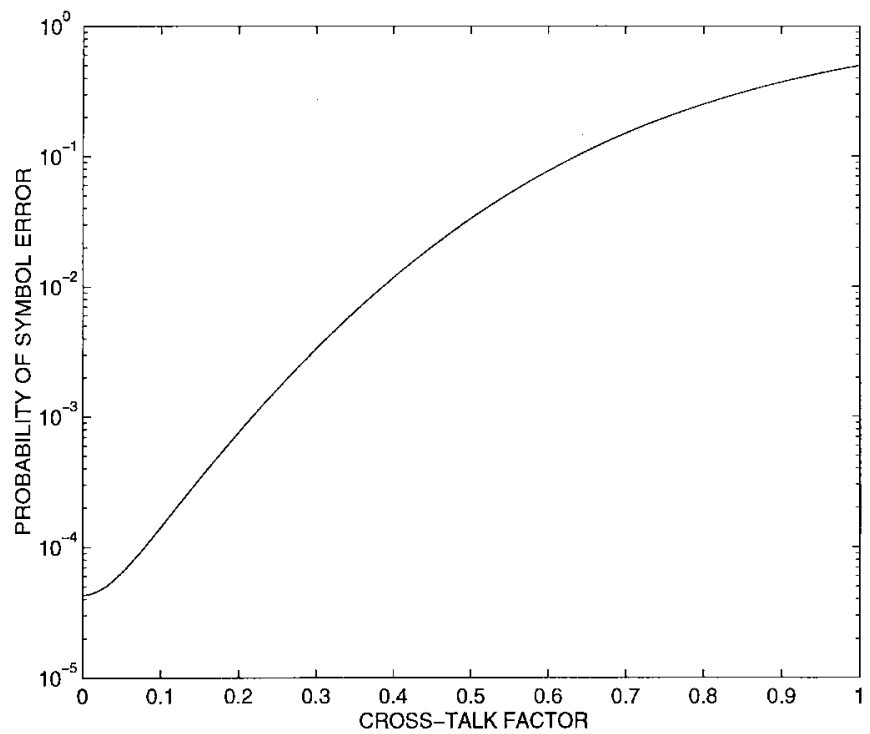

Fig. 1. QPSK $\widehat{P}_{\mathrm{E}}$ versus crosstalk factor $r$.

[3]. Because this approach has been shown to make certain analytical problems tractable, such as determining the effect of co-channel interference with signal fading [12], and signal jitter with ISI [13] on receiver performance, the method presented in this paper can extend these results to arbitrary modulation formats.

\section{REFERENCES}

[1] J. G. Proakis, Digital Communications, 3rd ed. New York: McGrawHill, 1995.

[2] C. W. Helstrom, "Calculating error probabilities for intersymbol and cochannel interference," IEEE Trans. Commun., vol. COM-34, pp. 430435, May 1986.

[3] N. C. Beaulieu, "The evaluation of error probabilities for intersymbol and cochannel interference," IEEE Trans. Commun., vol. 39, pp. 17401749, Dec. 1991.

[4] M. Nakhla, "Error probability for multilevel digital systems in presence of intersymbol interference and additive noise," IEEE Trans. Commun., vol. 42, pp. 2380-2383, July 1994.

[5] A. J. Levy, "Fast error rate evaluation in the presence of intersymbol interference," IEEE Trans. Commun., vol. COM-33, pp. 479-481, May 1985.

[6] O. Shimbo, R. J. Fang, and M. Celebiler, "Performance of $M$-ary PSK systems in Gaussian noise and intersymbol interference," IEEE Trans. Inform. Theory, vol. IT-19, pp. 44-58, Jan. 1973.

[7] K. Metzger, "On the probability density of intersymbol interference," IEEE Trans. Commun., vol. COM-35, pp. 396-402, Apr. 1987.

[8] P. J. Davis and P. Rabinowitz, Methods of Numerical Integration, 2nd ed. New York: Academic, 1984.

[9] D. E. Dudgeon and R. M. Mersereau, Multidimensional Digital Signal Processing. Englewood Cliffs, NJ: Prentice-Hall, 1984.

[10] A. Papoulis, The Fourier Integral and Its Applications. New York: McGraw-Hill, 1962.

[11] G. W. Stewart, Introduction to Matrix Computations. New York: Academic, 1973.

[12] N. C. Beaulieu and A. A. Abu-Dayya, "Bandwidth efficient QPSK in cochannel interference and fading," IEEE Trans. Commun., vol. 43, pp. 2464-2474, Sept. 1995.

[13] Z. Nikolic, M. Stefanovic, and N. Stojanovic, "Upper bound of error probability in the presence of intersymbol interference and jitter," Electron. Lett., vol. 30, no. 5, pp. 389-390, Mar. 1994. 